\title{
Seasonal variability in the incidence of carcinomatous meningitis
}

\author{
*Angelos Koutras, PhD,, Minas Sakellakis, MSc, ${ }^{1}$ Thomas Makatsoris, PhD, ${ }^{1}$ \\ Charalabia Psachoulia, BSc, ${ }^{3}$ Maria Kardari, BSc, ${ }^{3}$ Achilleas Nikolakopoulos, MSc, ${ }^{1}$ \\ Charalabos Gogos, PhD, ${ }^{2}$ and Haralabos P. Kalofonos, $\mathrm{PhD}^{1}$ \\ ${ }^{1}$ Division of Oncology, ${ }^{2}$ Department of Medicine, and ${ }^{3}$ Department of Pathology, University Hospital, Patras Medical School, Rion, \\ Patras, Greece
}

OBJECT The aim of the study was to investigate whether there are seasonal differences in the occurrence of carcinomatous meningitis $(\mathrm{CM})$, with a greater prevalence of the disease in months with higher temperatures.

METHODS The authors searched the records of all patients with a diagnosis of CM from 1998 until 2013 at the University Hospital of Patras, Greece. The date of hospitalization was extracted for each patient. The cases were divided into 2 categories depending on the time of $\mathrm{CM}$ diagnosis. Based on the official data regarding the annual temperature distribution in this region, the authors divided the patients into 2 groups. The first group consisted of cases diagnosed with CM from October 15 to April 15 (cold climate and shorter daytime duration), whereas the second group comprised patients diagnosed between April 15 and October 15 (warm climate and longer daytime duration).

RESULTS Overall, 44 confirmed cases of CM were found. The most common type of malignancy associated with the development of $\mathrm{CM}$ was breast cancer (27 patients), while the second most common tumor was lung carcinoma (11 patients). The median interval between the time of initial cancer diagnosis and CM was 4.5 years. Thirty-one patients were diagnosed with CM during the period between April 15 and October 15, while the remaining 13 patients developed CM between October 15 and April 15, a significant difference $(p=0.01)$.

CONCLUSIONS Significantly more patients developed CM during the warm season of the year. To the authors' knowledge, this is the first study to provide evidence for the potential seasonal variability in CM incidence. However, these results should be validated prospectively in larger cohorts.

http://thejns.org/doi/abs/10.3171/2014.11.JNS14174

KEY WORDS seasonal variability; leptomeningeal; metastasis; carcinomatous meningitis; melatonin; oncology

$\mathrm{D}$ ISSEMINATION of cancer cells in the CSF is an important issue in oncology because it is accompanied by profound clinical consequences. There are several ways in which cancer cells can invade the meninges and each of them is highly dependent on the histology of the primary tumor.

The incidence of clinically diagnosed leptomeningeal metastases is very low. However, asymptomatic cases are estimated to accompany at least $20 \%$ of solid tumors. . $^{10,11}$ There is evidence that the incidence of carcinomatous meningitis $(\mathrm{CM})$ has been increasing over the last decade. This increase is likely attributable to the progress in cancer therapeutics as well as the improvement in overall survival of cancer patients, providing time to the tumor to spread into the CNS. ${ }^{10,11}$ Chemotherapeutic agents generally have limited penetrance through the blood-brain barrier.

Breast and lung carcinoma, along with melanoma, represent the most prevalent causes of $\mathrm{CM}^{7}$ Despite the fact that leptomeningeal metastases can occur early in the course of the disease, $\mathrm{CM}$ generally represents a marker of disseminated disease, and overall prognosis is dismal. Preclinical data suggest that cancer progression can be influenced by circadian functions and may be associated with seasonal variability in outcome. ${ }^{1,4,13,21}$ However, no data regarding the seasonal variation in the incidence of $\mathrm{CM}$ have been reported so far. The aim of this study was to evaluate

ABBREVIATION CM = carcinomatous meningitis.

SUBMITTED January 24, 2014. ACCEPTED November 11, 2014.

INCLUDE WHEN CITING Published online January 9, 2015; DOI: 10.3171/2014.11.JNS14174.

DISCLOSURE The authors report no conflict of interest concerning the materials or methods used in this study or the findings specified in this paper.

* Drs. Koutras and Sakellakis contributed equally to this work. 
our clinical impression that there are seasonal differences in the occurrence of $\mathrm{CM}$, with a prevalence of the disease in months with higher temperatures and longer daytimes.

\section{Methods}

The study was conducted at the University Hospital of Patras, Greece. We searched the hospital records for all patients with a diagnosis of CM from 1998 until 2013, which was assigned either at admission or discharge. Fifty-five records were initially assigned, but a careful evaluation led to the rejection of 11 cases as ineligible for the study. Six of the cases were excluded because the diagnosis of CM was unclear. Four cases were excluded due to an underlying hematological malignancy, and the remaining case was rejected because the patient developed CM shortly after a cranial surgery. Consequently, 44 cases were available for further evaluation.

Parameters of interest included sex, age, type of underlying tumor, and time of first diagnosis. The primary end point of this retrospective analysis was to investigate whether a seasonal variability in the incidence of CM exists. For this reason, the date of hospitalization was extracted for each patient. The cases were divided into 2 categories depending on the time of CM diagnosis. The first group comprised patients diagnosed with $\mathrm{CM}$ during the cold months of the year. Based on the official existing data regarding the annual temperature distribution in our region, we concluded that the optimal 6-month interval that can be considered as a cold interval includes the period from October 15 to April 15 (Fig. 1). The climate in our region in this period of the year is quite harsh, especially in high altitude regions. However, there are not significant difficulties in accessing the hospital system during this time that might have influenced our results. Additionally, this period happens to be the period with a shorter daytime duration. The second group included cases diagnosed between April 15 and October 15, a period usually characterized by a warm climate and longer daytime duration.

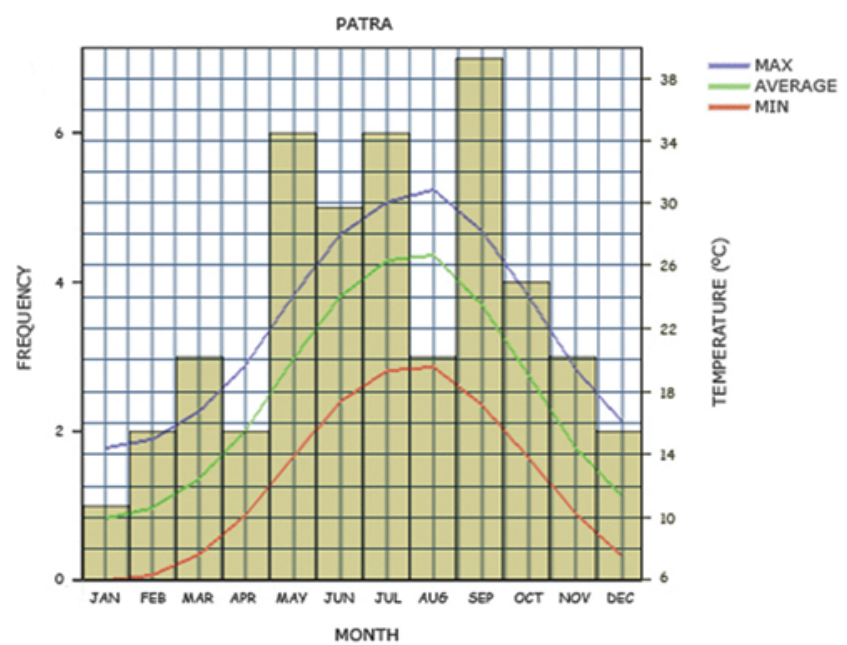

FIG. 1. Graph of the annual minimum, maximum, and average temperatures in the region of Patras, Greece (colored lines), combined with the annual distribution of diagnosed cases of CM (bars). Reprinted with permission from the Hellenic National Meteorological Service. Source: www.hnms.gr. Figure is available in color online only.
Data were evaluated using a binomial analysis. Statistical analysis was performed using IBM SPSS software.

\section{Results}

Definitive criteria for the diagnosis of $\mathrm{CM}$ included identification of neoplastic cells in the CSF or radiological findings suggestive of CM. However, we only included patients with a known prior history of cancer, either during treatment or during a surveillance program in our institution. Overall, 44 patients were diagnosed with CM. Among them, 40 cases were diagnosed on the basis of CSF analysis, while the remaining 4 had only radiological evidence. Thirty-three patients were women, while 11 were men $(\mathrm{p}=0.001)$. The median age for women was 51 years, while the median age for men was 47 years. The median interval between the time of initial diagnosis and $\mathrm{CM}$ was 4.5 years. The most common type of malignancy associated with CM in our study was breast carcinoma (27 cases). Among these patients, the median interval between the initial diagnosis and admission to the hospital was 5.5 years. The second most common tumor was lung cancer (11 patients). Nine of these patients had been diagnosed with a non-small cell carcinoma, whereas 2 had a smallcell lung cancer. The median time between initial diagnosis and the development of CM was 3 years. The remaining cases consisted of 2 patients with colorectal cancer, 2 cases with pancreatic cancer, 1 with gastric cancer, and 1 with ovarian cancer. All patients except for 2 developed $\mathrm{CM}$ while they had already been diagnosed with metastatic disease.

Thirteen patients developed CM during the period between October 15 and April 15, whereas the remaining 31 cases fell into the second group. Although the overall sample is relatively small, significantly more patients (31 vs $13, \mathrm{p}=0.01$ ) developed $\mathrm{CM}$ during the warm season of the year. The median age was 57 years for cases diagnosed during the cold months and 47 years for those in the warmmonth period $(p=0.1)$. Overall, 5 patients were diagnosed during winter, 11 during spring, 14 during summer, and 14 during autumn. Most patients were diagnosed during September ( 7 patients), followed by May (6 patients) and July (6 patients; Fig. 2). With respect to breast cancer, 19 patients were diagnosed during the warm period compared with 8 during the cold period $(\mathrm{p}=0.087)$. Regarding the sex of the sample, 23 women and 8 men were diagnosed in the warm period, compared with 10 and 3 in the cold period, respectively $(\mathrm{p}=0.035$ for women and $\mathrm{p}=0.225$ for men).

\section{Discussion}

To our knowledge, this is the first study to provide evidence for the possible seasonal variability in $\mathrm{CM}$ occurrence. However, evidence for circadian and seasonal effects in cancer progression already exists in the literature. ${ }^{4,21} \mathrm{Oh}$ et al. reported that the incidence of breast cancer declined during summer and winter, rather than being distributed randomly throughout the year. ${ }^{16}$ Furthermore, it was found that the metastatic behavior of breast cancer can follow specific patterns, with periods of inactivity in which new tumor colonies in the axilla are not being 


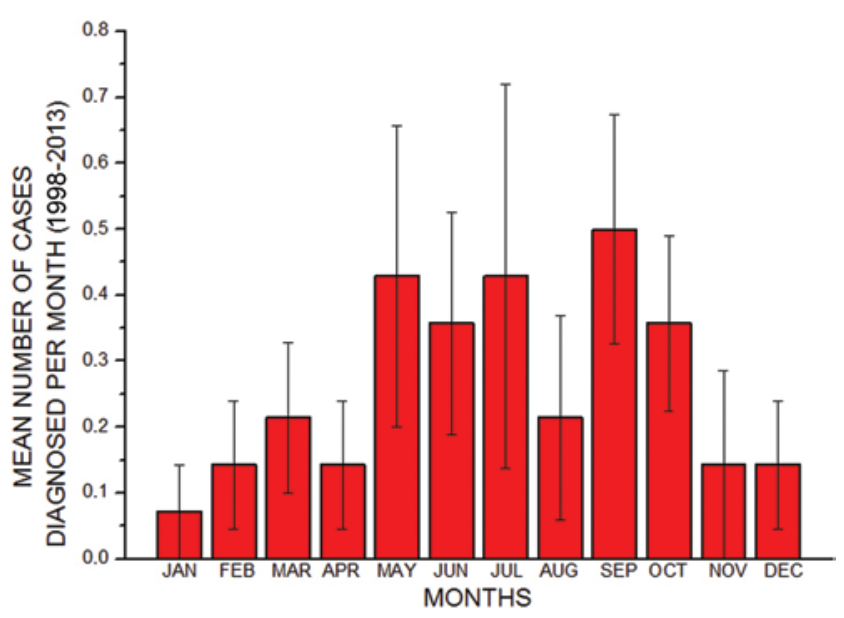

FIG. 2. Annual distribution of the mean number of diagnosed cases of $\mathrm{CM}$ per month with associated confidence intervals. Figure is available in color online only.

recruited. ${ }^{8}$ It was also shown that the rate of micrometastases in the axillary lymph nodes was higher during the second half of the year. ${ }^{9}$ Additionally, Mason et al. demonstrated that the season the initial tumor was diagnosed was highly associated with the prognosis in breast cancer models. Those who were diagnosed during spring or summer had a significantly longer overall survival, and this was independent of tumor size, nodal status, or hormone receptor status. ${ }^{13}$ Although the above-mentioned findings are not in complete accordance with our results, the fact that a seasonal variation may exist raises intriguing questions that need to be answered.

In our study we found a significantly increased incidence of CM during the warm months of the year in Greece. However, it is not clear whether it is the increased temperature of these months or the length of the daytime photoperiod that may play the most critical role. Several studies have demonstrated the influence of circadian factors on tumor growth rates, as well as the effect on the tolerability and response to antitumor agents. ${ }^{4,17,21}$ These cellular clocks are known to be influenced by factors such as the level of sunlight and the duration of the day, or factors affected by the season. In addition, there are molecular pathways such as the RANKL/RANK that are involved in thermoregulation and also appear to play a role in the metastatic process. ${ }^{5,6}$

Moreover, seasonal fluctuations in female hormone production, as well as known fluctuations in other hormones such as prolactin or melatonin, may represent additional factors contributing to the tendency of breast cancer to grow faster during the summer months, and the increased incidence of CM. It has been found in animals that short day exposure causes an alteration in the levels of steroid hormones, as well as an increase in melatonin secretion. ${ }^{15}$ One the other hand, prolonged treatment with melatonin mimics days of short duration..$^{15}$ All the abovementioned hormones can regulate immune functions and may influence the development of immune-related diseases, including cancer. ${ }^{3,15}$ Furthermore, anything that interacts with the photoperiod (such as ambient temperatures) potentially affects the function of the immune system.
The effect of melatonin has been confirmed in studies showing that increased levels are associated with a significantly lower risk for developing cancer. ${ }^{14,15}$ Melatonin downregulates gonadal hormones, influencing the growth rates of hormone-dependent tumors. ${ }^{19}$ Additionally, it can act as a naturally occurring antiestrogen..$^{19}$ Melatonin is also known to cause local effects in the brain parenchyma and its surroundings and can alter the vulnerability and permissiveness to tumor invasion. ${ }^{18}$ Melatonin can also provide antitumor effects by decreasing cell proliferation and inducing apoptosis. ${ }^{20}$ In addition, it has been shown in animal models of breast cancer that melatonin can carry antiangiogenic effects via downregulation of vascular endothelial growth factor expression. ${ }^{2}$ Moreover, it can also act synergistically with anticancer drugs, enhancing their effectiveness while reducing their side effects. ${ }^{12}$

Despite the fact that evidence for circadian and seasonal effects on cancer progression exist, thus far there are no definitive pathogenetic mechanisms for this observation. Consequently, theories that lead in this direction remain highly speculative. To the best of our knowledge, this is the first study reporting on the seasonal variability of CM incidence. A limitation of our analysis is the relatively small sample of patients, as well as the retrospective nature of the study.

\section{Conclusions}

In the present study we found a periodicity in the incidence of $\mathrm{CM}$ with a higher incidence during the warmer months of the year. However, our results should be considered hypothesis generating and should be validated prospectively in larger cohorts.

\section{References}

1. Akslen LA, Karwinski B, Maartmann-Moe H, Moberg I, Svendsen E, Hartveit F: Periodicity in metastatic tumour growth demonstrated in the human liver at autopsy. Preliminary report. Invasion Metastasis 11:58-64, 1991

2. Alvarez-García V, González A, Alonso-González C, Martínez-Campa C, Cos S: Antiangiogenic effects of melatonin in endothelial cell cultures. Microvasc Res 87:25-33, 2013

3. Brackowski R, Zubelewicz B, Romanowski W, Lissoni P, Barni S, Tancini G, et al: Preliminary study on modulation of the biological effects of tumor necrosis factor-alpha in advanced cancer patients by the pineal hormone melatonin. J Biol Regul Homeost Agents 8:77-80, 1994

4. Filipski E, King VM, Li X, Granda TG, Mormont MC, Claustrat B, et al: Disruption of circadian coordination accelerates malignant growth in mice. Pathol Biol (Paris) 51:216-219, 2003

5. Hanada R, Hanada T, Sigl V, Schramek D, Penninger JM: RANKL/RANK-beyond bones. J Mol Med (Berl) 89:647656, 2011

6. Hanada R, Penninger JM: [Central regulation of body temperature by RANKL/RANK pathway.] Clin Calcium 21:1201-1208, 2011 (Jpn)

7. Harstad L, Hess KR, Groves MD: Prognostic factors and outcomes in patients with leptomeningeal melanomatosis. Neuro Oncol 10:1010-1018, 2008

8. Hartveit F: Breast carcinoma: periodicity in presentation of metastatic tumour growth in the axilla. Clin Exp Metastasis 10:329-336, 1992

9. Hartveit F: Micrometastases to the axilla in breast cancer: 
their size and season of presentation. Invasion Metastasis 16:144-149, 1996

10. Kokkoris CP: Leptomeningeal carcinomatosis. How does cancer reach the pia-arachnoid? Cancer 51:154-160, 1983

11. Le Rhun E, Taillibert S, Chamberlain MC: Carcinomatous meningitis: leptomeningeal metastases in solid tumors. Surg Neurol Int 4 (Suppl 4):S265-S288, 2013

12. Lissoni $\mathrm{P}$, Barni $\mathrm{S}$, Mandalà $\mathrm{M}$, Ardizzoia $\mathrm{A}$, Paolorossi $\mathrm{F}$, Vaghi M, et al: Decreased toxicity and increased efficacy of cancer chemotherapy using the pineal hormone melatonin in metastatic solid tumour patients with poor clinical status. Eur J Cancer 35:1688-1692, 1999

13. Mason BH, Holdaway IM, Stewart AW, Neave LM, Kay RG: Season of initial discovery of tumour as an independent variable predicting survival in breast cancer. Br J Cancer 61:137-141, 1990

14. Nelson RJ, Demas GE: Seasonal changes in immune function. Q Rev Biol 71:511-548, 1996

15. Nelson RJ, Drazen DL: Melatonin mediates seasonal changes in immune function. Ann N Y Acad Sci 917:404-415, 2000

16. Oh EY, Ansell C, Nawaz H, Yang CH, Wood PA, Hrushesky WJ: Global breast cancer seasonality. Breast Cancer Res Treat 123:233-243, 2010

17. Ray M, Rogers LQ, Trammell RA, Toth LA: Fatigue and sleep during cancer and chemotherapy: translational rodent models. Comp Med 58:234-245, 2008

18. Reiter RJ, Acuña-Castroviejo D, Tan DX, Burkhardt S: Free radical-mediated molecular damage. Mechanisms for the protective actions of melatonin in the central nervous system. Ann N Y Acad Sci 939:200-215, 2001

19. Sánchez-Barceló EJ, Cos S, Fernández R, Mediavilla MD:
Melatonin and mammary cancer: a short review. Endocr Relat Cancer 10:153-159, 2003

20. Zhang S, Qi Y, Zhang H, He W, Zhou Q, Gui S, et al: Melatonin inhibits cell growth and migration, but promotes apoptosis in gastric cancer cell line, SGC7901. Biotech Histochem 88:281-289, 2013

21. Zorzet S, Perissin L, Rapozzi V, Giraldi T: Seasonal dependency of the effects of rotational stress and cyclophosphamide in mice bearing lewis lung carcinoma. Brain Behav Immun 16:368-382, 2002

\section{Author Contributions}

Conception and design: Sakellakis, Koutras. Acquisition of data: Sakellakis, Psachoulia, Kardari. Analysis and interpretation of data: Sakellakis, Koutras, Kardari. Drafting the article: Sakellakis, Koutras, Makatsoris, Kalofonos. Critically revising the article: all authors. Reviewed submitted version of manuscript: Koutras, Nikolakopoulos, Gogos, Kalofonos. Approved the final version of the manuscript on behalf of all authors: Sakellakis. Statistical analysis: Sakellakis, Koutras. Administrative/technical/material support: Makatsoris, Psachoulia, Nikolakopoulos. Study supervision: Koutras, Kalofonos.

\section{Correspondence}

Minas Sakellakis, Department of Oncology, University Hospital of Patras, Rion 26504, Greece. email: doctorsakellakis@gmail. com. 